\title{
Implementing the Royal College of Psychiatrists' guidelines for the practice of electroconvulsive therapy
}

\author{
AIMS AND METHOD \\ To implement the Royal College of \\ Psychiatrists' guidelines for the prac- \\ tice of electroconvulsive therapy \\ (ECT) and measure the impact of such \\ implementation on the quality of \\ treatment. \\ Treatment of ECT for periods of six \\ months before and after implemen- \\ tation of the guidelines were \\ studied. The effect on producing
}

\author{
adequate treatments and the actions \\ taken by the administrating doctor \\ were noted.

\section{RESULTS} \\ Once the guidelines were in place \\ there was a slight improvement in the \\ proportion of treatments considered \\ therapeutic and the doctor was more \\ likely to take appropriate action fol- \\ lowing an inadequate treatment.
}

\author{
However, it was also clear that \\ despite the guidelines being avail- \\ able they were not followed in a sig- \\ nificant number of instances.

\begin{abstract}
CONCLUSIONS
Implementation of the guidelines is siderable effort to ensure they are used. When they are followed there is an improvement in the practice of ECT.
\end{abstract} \\ not easy and there needs to be con-
}

It has been shown that psychiatrists have the lowest standards across the disciplines involved in the practice of electroconvulsive therapy (ECT) (Pippard \& Ellam, 1981; Pippard, 1992). The Royal College of Psychiatrists' audits also showed that, given the low standards in 1981, improvements by 1998 were modest, with only one-third of clinics meeting the College standards (Duffett \& Lelliot, 1998).

We wished to study the standard of the psychiatrist's practice of ECT within a unit before and after the introduction of a protocol in line with the College's guidelines (Royal College of Psychiatrists, 1984).

The study was completed in 1997 within Southport and Formby Community NHS Trust, which has a catchment area of approximately 130000 people. Within the service it had been assumed that the practice of ECT was adequate largely because of the apparent clinical efficacy and the low number of complaints from patients or professionals.

Psychiatric and vocational general practice trainees undertook the psychiatrist's duties for ECT, but as the regional training scheme incorporates many hospitals, no single centre has been responsible for their training in this area. ECT may therefore be administered without sufficient formal training or experience in local equipment and procedures. Prior to the audit there was no protocol in place for ECT and the study set out to identify whether introducing one and arranging training sessions for the trainees would improve practice.

\section{The study}

For a period of six months before and after the introduction of the training sessions, protocol and a recommended ECT machine (a Mectra SR2), all courses of ECT were studied. Patients were included in the study regardless of the number of treatments given or diagnosis, but they were excluded if their treatment course crossed the transition period.

Dose titration (to identify at the start treatment the minimum electrical stimulation required to produce a fit in an individual patient) was introduced and guidelines stated which treatments should be considered therapeutic and what action should be taken if they were not. Fit duration was used as an objective measure of likely efficacy, with a therapeutic duration taken as 20 seconds or more. The figure of 20 seconds was chosen as it is a little over the College's guideline of 15 seconds and thus should increase the likelihood of a therapeutic treatment. The upper end of the target range was taken as 50 seconds in an attempt to reduce cognitive side-effects. The duration of fit was measured visually using the cuff technique as only the Mectra SR2 had an electroencephalogram (EEG) facility.

The initial training session concentrated on familiarity with the protocol and method of dose delivery. Six subsequent sessions were then supervised by the trainer (a senior registrar with an interest in the area) to ensure the guidelines were being followed. The course was taken by all psychiatrists who would be administering ECT and included discussion with other participating staff. 
Table 1. Results of treatment before and after implementation of guidelines

original papers

Changes to administration

\begin{tabular}{|lll} 
& \multicolumn{2}{l}{ Changes to administration } \\
\cline { 2 - 3 } & Before & After \\
\hline$<20$ secs & $(79 / 213) 37 \%$ & $(21 / 132) 16 \%$ \\
$20-50$ secs & $(131 / 213) 62 \%$ & $(92 / 132) 70 \%$ \\
$>50$ secs & $(1 / 213)<1 \%$ & $(16 / 132) 12 \%$ \\
Not recorded & $(2 / 213) 1 \%$ & $(3 / 132) 2 \%$ \\
$\begin{array}{l}\text { Non-therapeutic treatments } \\
\text { restimulated }\end{array}$ & $(5 / 81) 6 \%$ & $(12 / 24) 50 \%$ \\
\hline
\end{tabular}

The introduction of a recommended ECT machine allowed a greater range of doses to be given at both extremes and incorporated an EEG reading, which gave a second measure of fit duration (although this was not used in comparison of data). Thus the following variables were changed as part of the audit:

(a) introduction of protocol and training session;

(b) supervision for six sessions;

(c) use of dose titration technique;

(d) profile of ECT raised within the department;

(e) new ECT machine (Mectra SR2 in place of Ectron 5);

(f) different patients treated;

(g) different doctors giving the treatment.

\section{Findings}

Before and after the change in practice, the patients were of similar average age (70 and 68 years respectively) and a similar number of courses of treatment were completed (18 and 14). There was no significant difference in the number of treatments in each course. Despite similar average starting doses (both approximately $275 \mathrm{mC}$ ), there was a markedly greater range when the protocol was in place, owing to the introduction of dose titration. There were fewer sub-therapeutic treatments with the protocol in place (see Table 1) and this may be due to the increase in frequency of restimulation when fits were shorter than 20 seconds.

Even after the introduction of a protocol, following an excessively short or long fit, appropriate changes to the charge were not usually made in the next session. However, when changes in line with the protocol were made (by adjusting the dose in the next session) the outcome was usually an 'ideal range' fit, thus supporting the validity of the protocol.

\section{Discussion}

From the results above, it seems that improvements have occurred in some areas of practice, but not all. There was a reduction in assumed sub-therapeutic treatments. However, there was also an increase in prolonged ( $>50$ seconds) fits which may be associated with a greater risk of side-effects. Following the introduction of guidelines, restimulation was more likely to be carried out if indicated, although this was still in only half of cases (see Table 1). When the fit duration fell outside the target of 20-50 seconds, appropriate changes of dose in subsequent sessions were more likely with the protocol in place, but again not usual.

It is therefore suggested that the introduction of changes described would result in patients being more likely to receive a therapeutic treatment. However, these improvements were not to the standard of the protocol itself and so could be greater.

The audit is concerned with the various changes to ECT administration in order to follow the guidelines suggested by the Royal College of Psychiatrists as it is implied that introduction of, and training in, a protocol can lead to improved practice.

If the suggestion that ECT is more effective with fit duration over 20 seconds is correct, then attempting to follow the College guidelines is more likely to lead to effective treatment (sub-therapeutic fits fell from $37 \%$ to $16 \%)$. However, as it was fit duration rather than clinical improvement that was measured, what cannot be seen from this study is whether treatment with ECT was actually more efficacious. It is generally accepted that clinical response within a treatment course, as much as fit duration, should determine the dose to be given. In addition, with several changes to practice having to be undertaken at one time it is not clear to what extent, if any, each of the measures affected the overall outcome.

Reviews of ECT practice (Pippard \& Ellam, 1981; Pippard, 1992; Duffett \& Lelliott, 1998) have raised awareness of the problem of inconsistent and, in places, poor practice. Despite this, two-thirds of clinics did not have a written stimulation policy for psychiatrists (Duffett \& Lelliott, 1998).

What this study has shown is that even in a service where the department is apparently functioning well, ECT administration can be improved. Furthermore, this improvement could be greater if the guidelines were more consistently followed, and it is clear that efforts to achieve this must go beyond education and initial supervision.

\section{References}

DUFFETT, R. \& LELLIOTT, P. (1998) the College. British Journal of Auditing electroconvulsive therapy. The Psychiatry, 139, 563-568. third cycle. British Journal of Psychiatry, 172, 401-405.

PIPPARD, J. (1992) Audit of electroconvulsive treatment in two NHS regions. British Journal of Psychiatry, 160, 621-637.

— \& ELLAM, L. (1981) Electroconvulsive ROYAL COLLEGE OF PSYCHIATRISTS (1994) ElectroconvulsiveTherapy (ECT). The Official VideoTeaching Pack of the Royal College of Psychiatrists Special Committee on ECT. London: Royal College of treatment in Great Britain: a report to

*Guy Brookes Specialist Registrar in General Adult Psychiatry, Airedale General Hospital, West Yorkshire (Ingrow Community Mental HealthTeam, 2000 South Street, Keighley, West Yorkshire BD21 1BB), John Rigby Medical Director, Hesketh Centre, Southport, Merseyside, Richard Barnes Consultant in Old Age Psychiatry, Ormskirk General Hospital, Lancashire 